\title{
Use of the AGobi All-in-One Airway Stabilizing Device During Fiberoptic Bronchoscopy: A Case Study Report
}

Ghobrial VG*, Schon A and Childress D

Associates/Respiratory Medicine, Bradenton, Florida, USA

\begin{abstract}
Airway management during endoscopy is highly critical, and this is particularly true in older patients with complex medical issues. Over the course of the last few decades, tremendous advances in various airway stabilization devices used during endoscopic procedures have occurred. This includes not only laryngeal masks but a variety of extraglottic devices as well. Identifying airway stabilization devices that offer patient safety, patient comfort and ease-of-use while promoting high quality results remains a continual process. With this in mind, a case study presentation is provided involving a complicated elderly patient undergoing fiberoptic bronchoscopy that utilized the AGobi All-inOne Airway Stabilization device. The case experience will highlight the advantages of this supraglottic airway device while an examination of the research literature will discuss its potential limitations. Based on this case study, the use of this device in fiberoptic bronchoscopy and in a number of other situations where airway stabilization is needed is supported. In addition, the case study also supports further study of the AGobi All-in-One Airway Stabilizing device in comparison to other airway stabilizing options in specific patient scenarios.
\end{abstract}

Keywords: Airway stabilization device; Bronchoscopy; Supraglottic airway device; Endoscopy; AGobi airway device; Case study

\section{Introduction}

Airway stabilization is required for various patient care situations ranging from emergent to routine. Likewise, effective airway control and maintenance reflect essential aspects of respiratory, anesthetic, and gastroenterology specialties during specific procedural care [1]. Various devices have been employed to this extent, and in recent decades, this has included extra-glottic airway devices (EAD) or supra-glottic airway devices (SGA) [2]. Those developed in the 1980s and 1990s were labeled first generation; those in the 2000s were labeled second generation; and more recent ones termed third generation [2]. While some clarifications are needed regarding the purpose and features of these devices, successive generations tend to have enhanced comfort and safety features for the patient [2].

The need for more advanced airway stabilizing devices stems from a number of challenges that healthcare specialists face when trying to stabilize patient airways. Some devices require excessive force for placement and carry risks for airway trauma. Placement for some devices often requires multiple attempts, and a variety of operators may need to be trained on device placement [3]. Other issues relate to the multiple purposes for which the devices are used. Lastly, several devices fail to provide adequate visualization of related airway structures, particularly for those specialists performing endoscopic procedures [3].

From this perspective, the following provides a case study report of a complex patient undergoing fiberoptic bronchoscopy with the aid of a new, third generation, airway-stabilizing device with advanced features. The device, the AGobi All-in-One Airway Stabilization device, is unique in that it provides multiple ports to serve multiple functions, and it offers several amenities that enhance patient comfort, reduce risks of trauma, and allow ease-of-use by multiple providers. The device does not extend past the vocal cords and/or the larynx, and based on its anatomical positioning, the device remains in an extra-glottic or supraglottic location. The device, however, does not offer any seal or obliteration of the pharynx while used. Given the lack of a pharyngeal seal, the device will be described as an airway-stabilizing device with advanced features as a result.

In an effort to highlight these advantages, a case study using this airway-stabilizing device with advanced features will be provided. In addition, key features of this device and its potential advantages and limitations will be discussed. The purpose of this case study is thus to introduce this device and its successful application in complex patients during endoscopic procedures. Likewise, the case study serves to show the need for additional research studies comparing the device's efficacy in relation to other airway stabilizing device options.

\section{Case Study Presentation}

The case study being presented involves an 82-year-old Caucasian man with known hypertension, hypercholesterolemia, atrial fibrillation and obstructive sleep apnea, who originally presented to an acute care hospital in Florida in early 2018. Presenting symptoms included sudden onset neck and chest pain, which was subsequently confirmed to be secondary to an acute myocardial infarction. Complications of the patient's heart attack included the development of sudden cardiac arrest, which required emergent coronary artery bypass grafting (CABG) and intra-aortic balloon pump support. Postoperatively, the patient required persistent mechanical ventilation support resulting in the eventual placement of a tracheostomy tube. Additional complications also included acute renal failure that required hemodialysis support as well as poor enteral nutrition that necessitated percutaneous gastrostomy tube placement.

Because of the patient's numerous complications and need for longterm support, the patient was eventually transferred to a long-term acute care facility in April, 2018. Pulmonary specialty assessment was performed at that time. The patient was relatively stable then with mild

*Corresponding author: Victor G. Ghobrial, Associates/Respiratory Medicine Bradenton, Florida, USA, Tel: (941) 746-7444; Fax: (941) 746-1777; E-mail: victorghobrial@ymail.com

Received August 23, 2018; Accepted September 12, 2018; Published September 18, 2018

Citation: Ghobrial VG, Schon A, Childress D (2018) Use of the AGobi All-in-One Airway Stabilizing Device During Fiberoptic Bronchoscopy: A Case Study Report. J Pulm Respir Med 8: 472. doi: 10.4172/2161-105X.1000472

Copyright: $\odot 2018$ Ghobrial VG, et al. This is an open-access article distributed under the terms of the Creative Commons Attribution License, which permits unrestricted use, distribution, and reproduction in any medium, provided the original author and source are credited. 
bilateral anterior ronchi, persistent renal failure requiring hemodialysis, and continued mechanical ventilator dependency via tracheostomy tube. In addition to routine pulmonary and tracheostomy site care, recommendations were made to continue ventilator weaning, fluid management, and respiratory monitoring with bronchodilator use. Likewise, continued cardiac and renal management supports were encouraged.

A few weeks later, pulmonary re-consultation was requested due to inability to progressively wean the patient from mechanical ventilation and due to the presence of mild dyspnea. The decision to perform fiberoptic bronchoscopy was made, and after informed consent was obtained, the procedure was performed under intravenous sedation. The tracheostomy tube was capped during upper airway evaluation. The patient required supplemental oxygen, which was provided via his tracheostomy tube using a heat moisturizing exchange (HME) device. The initial portion of the fiberoptic bronchoscopy was completed via the tracheostomy site where the carina, right and left bronchi, and all lobes of both lungs were visualized. Likewise, bronchial washings were collected from all lung regions into a sterile side trap. No pathologies were identified during this portion of the procedure.

Subsequently, the AGobi All-in-One Airway Stabilizing device was introduced through the oral cavity with the aid of a lubricant. The device was inserted by depressing it against the tongue and allowing it to easily slide into place. The device's rubber straps were then tightened to secure the device in place around the patient's head for the procedure. In one of the two lateral ports of the device, supplemental oxygen was administered, and in the other lateral port, an end-tidal $\mathrm{CO}_{2}$ monitor was placed. The fiberoptic bronchoscope was then introduced through the central port and switched intermittently with a suctioning device to enhance visualization when respiratory secretions needed clearing.

During the fiberoptic bronchoscopy portion through the oral cavity, the epiglottis and vocal cords were noted to be normal anatomically with normal vocal cord coaptation during phonation and respirations. In the sub-glottic region, the tracheostomy tube was in good position, and granulation tissue was present without inflammation. However, during expiration, the membranous portion of the trachea came in close proximity with the granulation tissue resulting in a $75 \%$ reduction of airway patency in the antero-posterior diameter. The patient tolerated the procedure well without complication. Findings were reported to primary care physician with subsequent management plans arranged.

\section{Discussions}

In considering options regarding airway stabilization during endoscopic procedures, the potential devices available for use has recently increased. Classically, laryngoscopes were utilized originally since relatively few options existed. However, this was quickly replaced by laryngeal mask airways that allowed supraglottic airway stabilization while also enabling positive pressure ventilation and oxygenation [4]. While numerous laryngeal mask airway types now exist, additional supraglottic and infraglottic airway devices have also been introduced into the market. As noted before, these have been labeled according to different generations of models based on the decade that they were introduced [2].

Debate currently exists regarding the nomenclature of these newer airway stabilization devices. Some have suggested these should all be included as extra-glottic devices and distinguished based on specific features [2]. Others have suggested that categories should reflect whether or not intubation is permissible with the use of the device or not [5]. While the current case study does not seek to settle these debates, it does seek to detail specific features of one airway-stabilizing device with advanced features and its benefit-risk profile in an effort to raise awareness and to encourage additional study.

In the current case study, the use of the AGobi All-in-One Airway Stabilizing device provided several advantages. As an airway-stabilizing device with advanced features, it allowed simple airway stabilization with ease and was easily positioned into place during fiber optic bronchoscopy [6]. Likewise, the device's soft gel tip reduced the risk of oropharyngeal trauma, which is a frequent concern especially among older patients and individuals with upper airway pathologies. The locking rubber head strap permitted a secure positioning of the device as well, and the avoidance of subsequent positioning maneuvers also reduced risk of trauma. The device also provided a bite block that protected the patient's teeth, prevented closure onto the endoscopic tube, and facilitated comfort of the patient's jaw. Lastly, the ability to perform the bronchoscopy while administering oxygen, monitoring end-tidal $\mathrm{CO}_{2}$, and have ready access to suctioning further facilitated efficiency and safety during the procedure [1].

While these features of the AGobi All-in-One Airway Stabilizing device were notable in this particular patient, the device has additional benefits that were not highlighted in the case study. Specifically, the device is composed of high-grade, standardized materials, yet it is disposable and highly flexible in nature. The device also permits endotracheal intubation when using a $6 \mathrm{~mm}$ or smaller diameter endotracheal tube. The airway-stabilizing device with advanced features also has specialized flavor pads that provide enhanced taste aesthetics for the patient. Finally, the AGobi device does not require special training for placement instruction, which permits its placement by a variety of healthcare professionals in a number of settings [6].

In considering potential disadvantages of the AGobi All-in-One Air Stabilizing device, it is not ideal in every instance. For patients with high risk for aspiration, endotracheal tube placement is classically preferred. For example, patients with high risks for bleeding, fluid accumulation, or cyst rupture during such a procedure may be better served with greater airway protection precautions [7]. This device also has no protection from gastric reflux, which may be a concern for some patients undergoing gastric endoscopy procedures [3]. However, the device offers many additional comfort and safety features as noted over other bite blocks and other supraglottic airway stabilizing devices [3]. Given that over 60 percent of sedated anesthetic procedures utilize SGA devices, this particular device appears to be a nice addition to the armamentarium now available to endoscopic specialists [7].

\section{Conclusions}

The present case study involves an elderly male with numerous health conditions who underwent fiberoptic endoscopy to assess mild dyspnea and the inability to wean off tracheostomy-supported mechanical ventilation. The procedure was facilitated by the use of an AGobi All-in-One Airway Stabilizing device, which is best categorized as an airway-stabilizing device with advanced features relevant to patient safety, patient comfort, and enhanced airway stabilization and monitoring. The use of this airway-stabilizing device with advanced features facilitated efficiency and safety of patient care during this case study, and no complications or delays in patient recovery were experienced. Based on this case experience, and given the current state of affairs concerning device use during endoscopic procedures, further study regarding the AGobi All-in-One Airway Stabilizing device is supported based on case experience. 
Citation: Ghobrial VG, Schon A, Childress D (2018) Use of the AGobi All-in-One Airway Stabilizing Device During Fiberoptic Bronchoscopy: A Case Study Report. J Pulm Respir Med 8: 472. doi: 10.4172/2161-105X.1000472

\section{References}

1. Amin N, Sharma S (2017) Airways, connectors, laryngoscopes and nonrebreathing valves. Objective anaesthesia review: A comprehensive textbook for the examinees 440

2. Agro FE, Pascarella G (2018) Extraglottic airway devices: Is the classification in generations really informative of properties and safety. Minerva Anestesiol 84: 649-651.

3. Goudra B, Singh PM (2017) Airway management during upper GI endoscopic procedures: State of the art review. Dig Dis Sci 62: 45-53.

4. Van Zundert AAJ, Skinner MW, Van Zundert TCRV, Luney SR, Pandit JJ (2016) Value of knowing physical characteristics of the airway device before using it. Br J Anaesth 117: 12-16.

5. Cook TM (2015) Third generation supraglottic airway devices: an undefined concept and misused term. Time for an updated classification of supraglottic airway devices. Br J Anaesth 115: 633-634.

6. Heegeman DJ, Rosandick WD, Boehning-Anderson RH, Woltmann AR (2018) Supraglottic airway device placement by respiratory therapists. Am J Emerg Med pii: S0735-S6757.

7. Arevalo-Ludena J, Arcas-Bellas JJ, Alvarez-Rementería R, Flandes J, Morís $\mathrm{L}$, et al. (2016) A comparison of the I-Gel supraglottic device with endotrachea intubation for bronchoscopic lung volume reduction coil treatment. J Clin Anesth 31: 137-141. 